\title{
Aspectos de la reproducción de Plagioscion magdalenae (Pisces: Sciaenidae) en el embalse El Guájaro, bajo Magdalena, Colombia Reproduction aspects of Plagioscion magdalenae (Pisces: Sciaenidae) in El Guájaro reservoir, lower Magdalena, Colombia
}

\author{
René A. Rojas-Luna ${ }^{\square} \square$, Carlos A. García-Alzate ${ }^{\square} \square$
}

\section{Resumen}

Plagioscion magdalenae es el recurso íctico más importante del embalse El Guájaro, en el norte de Colombia, y para sus pobladores ribereños, fundamental para las pesquerías de subsistencia de la región, lo que ha llevado a su sobreexplotación. En este artículo se describen los aspectos reproductivos de P. magdalenae, en este embalse. Se realizaron diez muestreos de recolección mensuales, entre abril de 2018 y enero de 2019, con el fin de abarcar cada momento del pulso de inundación. Se calcularon la proporción sexual, relación gonadosomática (RGS), fecundidad, diámetro de ovocitos y talla media de madurez sexual $\left(\mathrm{L}_{50}\right)$. Se analizaron 207 ejemplares, y se establecieron siete intervalos de tallas; 126 individuos fueron machos y 81 hembras, con una proporción sexual de 1:1.6 (hembra-macho). La RGS indicó que este pez tiene varios picos reproductivos, los principales en julio y septiembre. La fecundidad absoluta fue 1909 ovocitos para aguas bajando, 1590 para aguas bajas, 2435 para aguas subiendo y 2132 para aguas altas, con un diámetro promedio de ovocito de $0.29 \mathrm{~mm}$. Los machos maduraron primero que las hembras y la $\mathrm{L}_{50}$ estuvo por debajo de la talla media de captura $(30 \mathrm{~cm})$, lo cual refleja que la normativa legal está bien determinada, pero no hay regulación para su pesca.

Palabras clave. Diversidad. Ecología. Pez neotropical. Reproducción.

\begin{abstract}
Plagioscion magdalenae is the most important fish resource of the El Guájaro reservoir, Colombia, and its riparian inhabitants, fundamental for subsistence fisheries in the region, which has led to its overexploitation. This paper describes and evaluates the reproductive aspects of $P$. magdalenae in this reservoir. Ten monthly collection samplings were made between April 2018 and January 2019, in order to cover each moment of the flood pulse. We calculated sex ratio, gonadosomatic ratio (RGS), fecundity, oocyte diameter and minimum and mean size at sexual maturity $\left(\mathrm{L}_{50}\right)$. We analyzed 207 specimens and established seven size intervals; 126 individuals were males and 81 were females, with an overall sex ratio 1:1.6 (female-male). The RGS indicated that this fish has several reproductive peaks, the main ones being in July and September. Absolute fecundity was 1909 oocytes for falling water, 1590 for low water, 2435 for rising water and 2132 for high water; with an average oocyte diameter of 0.29 $\mathrm{mm}$. Males matured earlier than females and their mean size at sexual maturity was below the mean catch size $(30 \mathrm{~cm})$, reflecting that legal regulations are well stipulated, but there is no strict control for fishing.
\end{abstract}

Key words. Diversity. Ecology. Neotropical fish. Reproduction. 


\section{Introducción}

En la cuenca del río Magdalena, la pácora, Plagioscion magdalenae (Steindachner, 1878), es una especie altamente explotada y representa un recurso pesquero de gran importancia para la población ribereña y para las principales ciudades costeras del país. En el embalse El Guájaro, esta especie es la primera en la pesca de subsistencia de los pobladores ribereños y está relacionada con la disminución en las capturas en los últimos años de otras especies de importancia pesquera de la cuenca como el bocachico (Prochilodus magdalenae), bagre rayado (Pseudoplatystoma magdaleniatum), capaz (Pimelodus grosskopfii) y nicuro (Pimelodus yuma), las cuales están desapareciendo (Lasso et al., 2011).

El género Plagioscion lo componen cinco especies válidas y ampliamente distribuidas en América del Sur (Souza et al., 2019). En Colombia, P. magdalenae se distribuye en las cuencas Caribe, Magdalena y Amazonas (Casatti, 2005; Maldonado-Ocampo et al., 2008; García-Alzate et al., 2020).

La reproducción de los peces, en las cuencas de ríos tropicales, generalmente es temporal y existe una sincronización entre los procesos reproductivos y el incremento en el nivel de las aguas ocasionado por el régimen hidrológico, actuando como indicador final del periodo reproductivo (Criscuolo, 2005; Pareja-Carmona et al., 2014). Con el aumento de la piscicultura en el país durante las seis últimas décadas, se han incrementado las investigaciones sobre la reproducción de peces continentales de importancia comercial (Merino, 2018). En la actualidad, P. magdalenae no cuenta con estudios poblacionales en el embalse El Guájaro, que permitan conocer su estado ni su capacidad de carga; por esta razón, se requieren análisis acerca de su ecología. Información acerca de los procesos reproductivos de esta especie de alto valor socioeconómico son relevantes para promover el uso sostenible y aprovechamiento adecuado del recurso, mediante procesos de ordenación de la pesca, evitando que la explotación supere la capacidad de carga de las poblaciones de la pácora, con el riesgo latente de extinciones locales (Cardoso et al., 2018). La sobrepesca en el bajo Magdalena sigue siendo la principal amenaza para esta especie, y los pescadores del embalse El Guájaro ejercen una gran presión sobre ella. Esto ha llevado a que la especie sea catalogada en estado de vulnerabilidad en la cuenca del Magdalena, y de casi amenazada a nivel nacional (Álvarez-León y Jiménez-Segura, 2012). El objetivo de este trabajo fue evaluar los aspectos de la reproducción de $P$. magdalenae en el embalse El Guájaro, cuenca baja del río Magdalena.

\section{Materiales y métodos}

Área de estudio. El área hidrográfica del Magdalena-Cauca (5 701101 ha) alberga los embalses más grandes del país, que representan el $18 \%$ del total de humedales continentales a nivel nacional. El embalse El Guájaro fue construido entre 1964 y 1965 por Instituto Colombiano de la Reforma Agraria (INCORA), con el fin de almacenar agua para los distritos de riego del sur del departamento del Atlántico (CRA, 2002). Se ubica en la cuenca baja del Magdalena, entre los $10^{\circ} 25^{\prime}$ y $10^{\circ}$ $38^{\prime} \mathrm{N}$ y $75^{\circ} 00^{\prime} 75^{\circ} 08^{\prime} \mathrm{W}$, y a 3-13 m s.n.m., en el margen izquierdo del Río Magdalena, al suroccidente del Departamento del Atlántico. Actualmente ocupa una extensión de 13000 ha, donde se desarrollan principalmente actividades pesqueras, agrícolas, recreación y turismo, siendo el cuerpo de agua más importante de la región (CRA, 2002; Caraballo, 2009; García-Alzate et al., 2016).

Fase de campo. Se realizaron diez muestreos mensuales de recolección, entre abril de 2018 y enero de 2019, con el fin de abarcar cada momento del pulso de inundación (Figura 1). La obtención de los peces se realizó con ayuda de los pescadores y pesquerías. Para cada individuo se evaluó el peso total (g) con una balanza analítica (Ohaus CS2000 $\pm 0.0001 \mathrm{~g}$ ), y la longitud total y estándar $(\mathrm{mm})$ con un calibrador digital $( \pm 0.01 \mathrm{~mm})$. Seguidamente, los peces fueron disectados con un corte uroventral para extraer estómagos, intestinos y gónadas. Cada una de estas estructuras fue pesada (g), medida (mm), fijadas en alcohol al 70 $\%$ y depositada en recipientes plásticos debidamente rotulados con la información pertinente del ejemplar (número de etiqueta y de ejemplar, fecha y lugar de recolección, sexo y estructura fijada; se sigue a Morales y García-Alzate (2016).

Fase de laboratorio. Se estableció la relación entre longitud total/peso total, mediante gráficas a partir de la ecuación de regresión potencial $\mathrm{WT}=\mathrm{a} \times \mathrm{LT}^{\mathrm{b}}$ (Sokal y Rohlf, 1987). Se consideró el crecimiento isométrico b $<3 \mathrm{u}$ alométrico b $>3$ (Froese, 2006). Los ejemplares se distribuyeron en tallas según su longitud estándar, a través de la ecuación: $A=N / R, R=\sqrt{ }$ y $N=$ Max-Min, donde $A=$ amplitud de los intervalos, $R=$ número de intervalos, $\mathrm{n}=$ número de observaciones, $\mathrm{Max}=$ valor máximo y Min=valor mínimo (Morales y García-Alzate, 2016). 


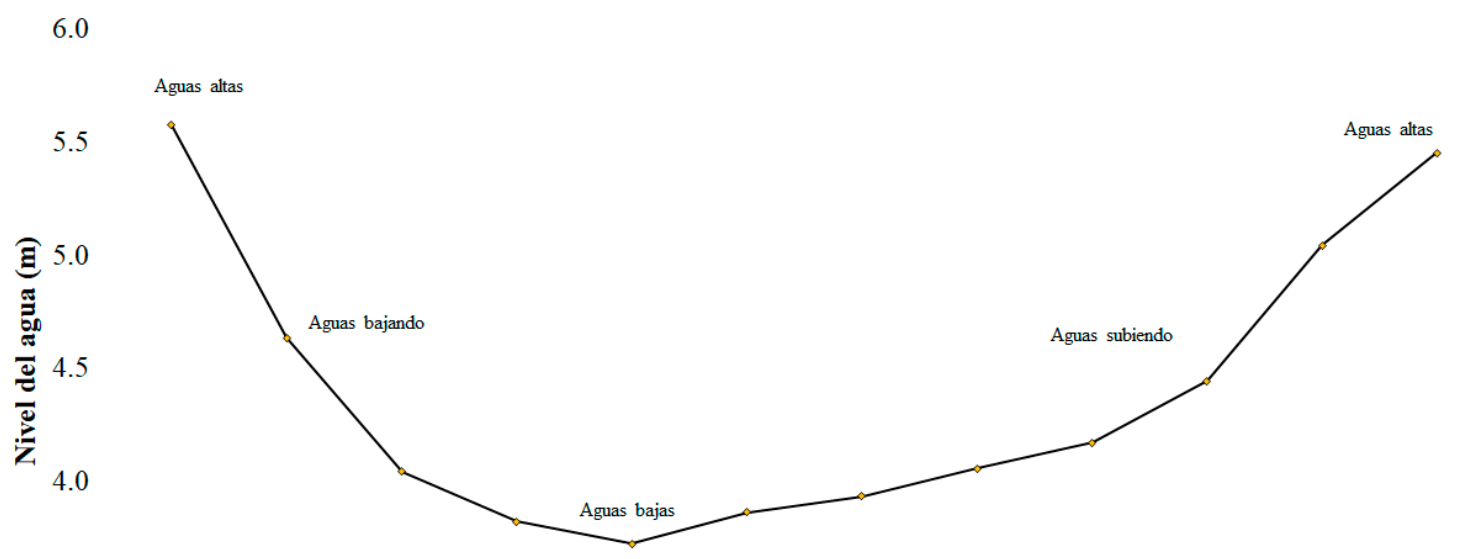

3.5

3.0

Ene Feb Mar Abr May Jun Jul Ago Sept ${ }^{\prime}$ Oct Nov Dic

Figura 1. Promedio histórico del nivel del agua del embalse El Guájaro, Colombia, de la estación AcuaCultivos (2004-2011), y pulso de inundación modificado de García-Alzate et al. (2016).

Figure 1. Historical mean water level of El Guájaro reservoir, Colombia from AcuaCultivos station (2004-2011) and flood pulse modified from García-Alzate et al. (2016).

Se calculó el factor de condición K (Vazzoler, 1996) para conocer el grado de robustez y bienestar del pez, que presupone un incremento alométrico del peso como función del tamaño del individuo, mediante la ecuación $\mathrm{K}=\mathrm{Wt} /$ $\mathrm{Le}^{\mathrm{b}}$, donde Wt es el peso total del ejemplar (g), Le es la longitud estándar $(\mathrm{mm})$ y $b$ es el coeficiente angular de la regresión peso total-longitud estándar.

Para estimar los aspectos reproductivos se determinó la proporción de sexos (Wenner, 1972) a partir de la ecuación: \% Machos $=100 *(\mathrm{Nm} / \mathrm{Nt})$, donde $\mathrm{Nm}=$ es el número de machos y $\mathrm{Nt}=$ el número total de individuos. La talla media de madurez sexual se determinó mediante el método estadístico gráfico, cuando más del 50 \% de la población entra en el período reproductivo (Sokal y Rohlf, 1995).

Las gónadas se preservaron en alcohol, luego se introdujeron por una semana en líquido Gilson modificado (Simpson, 1951), para así poder disolver la densa túnica albugínea que es característica de este tipo de especies. También se evaluó la relación gonadosomática (RGS) (Vazzoler, 1996), que indica la relación entre el avance de la maduración ovocitaria y el peso del cuerpo, y refleja el grado de desarrollo de la gónada; así: RGS=Wo/We*100, donde Wo es el peso de la gónada, We el peso del cuerpo $(\mathrm{Wt}-\mathrm{Wo} ; \mathrm{Wt}=$ peso total). La fecundidad $($ Ricker, 1968$)$ se estimó mediante el método de las sub-muestras secas, que consta de un secado en horno a $40^{\circ} \mathrm{C}$ durante 25 minutos, para el posterior conteo directo con una balanza analítica. Por último, con el fin de obtener el valor promedio de ovocitos en cada uno de los muestreos, se aplicó la ecuación para fecundidad absoluta $(\mathrm{Fa}): \mathrm{Fa}=$ $\Sigma \mathrm{n}^{\circ} / \mathrm{N}^{\mathrm{o}}$, donde $\mathrm{n}^{\mathrm{o}}$ es el número de ovocitos por hembra, y $\mathrm{N}^{0}$ el número total de hembras; la fecundidad relativa $(\mathrm{Fr})$ se calculó a partir de la fórmula $\mathrm{Fr}=\mathrm{Fa} / \mathrm{Wt}$, expresada como ovocitos por gramos de peso corporal total (Chaves y Vazzoler 1984; Araya et al., 2003), junto con su respectiva desviación estándar. El diámetro de ovocito se determinó según el método de submuestras secas (Ricker, 1968), e incluye desviación estándar (Ibarra-Trujillo y García-Alzate, 2017).

Análisis estadístico. El coeficiente $b$ de las ecuaciones de regresión potencial, se comparó entre sexos mediante una prueba T student, para indicar si existieron diferencias estadísticamente significativas (Barbosa et al., 2010). Para determinar el grado de asociación entre las relaciones de las variables longitud total y peso total, se utilizó el coeficiente de correlación de Pearson.

Además, se aplicó un test Chi-cuadrado para evaluar si existían diferencias estadísticamente significativas en cuanto a la proporción de sexos esperada y la registrada. 


\section{Resultados}

Se recolectaron 212 ejemplares, 129 machos $(60.9 \%)$ y 83 hembras (39.1\%), para una relación hembra/macho 1:1.6, sin diferencias significativas $\left(\chi^{2}: 8.07, p=0.05\right)$. Las hembras dominaron en septiembre, con 17 individuos $(8.2 \%)$, que coincide con el período de altas lluvias (aguas subiendo), y noviembre, con 12 ejemplares (5.8 $\%)$, que corresponde con la misma época; los machos fueron más abundantes principalmente en los meses de abril (aguas bajando) y enero (aguas altas), con 19 (9.2 $\%)$ y $20(9.7 \%$ ) ejemplares, respectivamente (Figura 2).

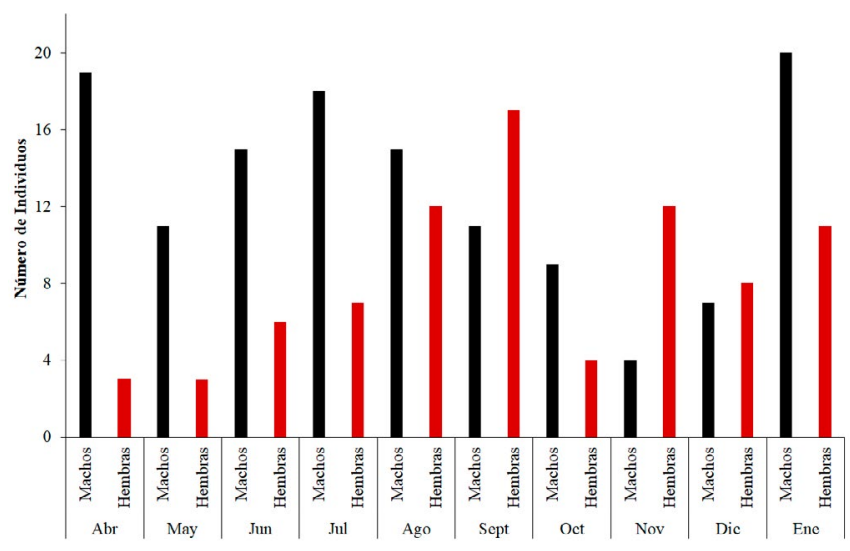

Figura 2. Número de individuos machos y hembras de Plagioscion magdalenae por meses, en el embalse El Guájaro, bajo Magdalena, Colombia.

Figure 2. Number of male and female individuals of Plagioscion magdalenae per month in El Guájaro reservoir, lower Magdalena River, Colombia.

La relación entre longitud total y peso total para machos, hembras y sexos agrupados fue altamente significativa $(\mathrm{p}<0.01)$ (Tabla 1). Se registró una alometría positiva para machos, hembras y sexos agrupados. No se encontraron diferencias estadísticamente significativas para el coeficiente de regresión $(b)$ entre machos y hembras $(t=12.70, p>0.05)$. Los individuos examinados presentaron longitudes entre 14.4 y 36.5 cm, con las longitudes más frecuentes entre 26.7 y 29.7 cm (talla V); por otro lado, el menor número de ejemplares se vio reflejado en la talla VII $(32.9-37.4 \mathrm{~cm})$ (Figura 3). La longitud máxima registrada fue de una hembra $(36.5 \mathrm{~cm})$; sin embargo, los individuos machos predominaron en todas las clases de talla, excepto en la talla VII, donde fue mayor la presencia de hembras. La talla media de maduración sexual en hembras fue de $27.0 \mathrm{~cm}$ de longitud estándar, mientras que en machos fue de $24.5 \mathrm{~cm}$ (Figura 4).

Tabla 1. Valores de la relación longitud total/peso total para machos, hembras y sexos agrupados de Plagioscion magdalenae en el embalse El Guájaro, bajo Magdalena, Colombia. n: Número de individuos; LT: Longitud total (mínimo y máximo); A: Constante de la ecuación de la regresión; b: Coeficiente de alometría de la ecuación de regresión; r: Coeficiente de correlación de Pearson; R2: Coeficiente de determinación de la gráfica de regresión potencial.

Table 1. Values of the total length/total weight relationship for males, females and grouped sexes of Plagioscion magdalenae in El Guájaro reservoir, lower Magdalena River, Colombia. N: Number of individuals; LT: Total length (minimum and maximum); A: Constant of the regression equation; b: Coefficient of allometry of the regression equation; r: Pearson correlation coefficient; $\mathrm{R}^{2}$ : Coefficient of determination of the potencial regression graph.

\begin{tabular}{lccccccc} 
& $\mathbf{n}$ & LT (min-max) & A & b & r & R2 & Alometría \\
Machos & 126 & $18.2-41.8$ & 0.0033 & 3.28 & 0.96 & 0.98 & + \\
Hembras & 81 & $19.5-43$ & 0.0024 & 3.38 & 0.96 & 0.98 & + \\
Sexos agrupados & 207 & $18.2-43$ & 0.0028 & 3.33 & 0.96 & 0.98 & + \\
\hline
\end{tabular}




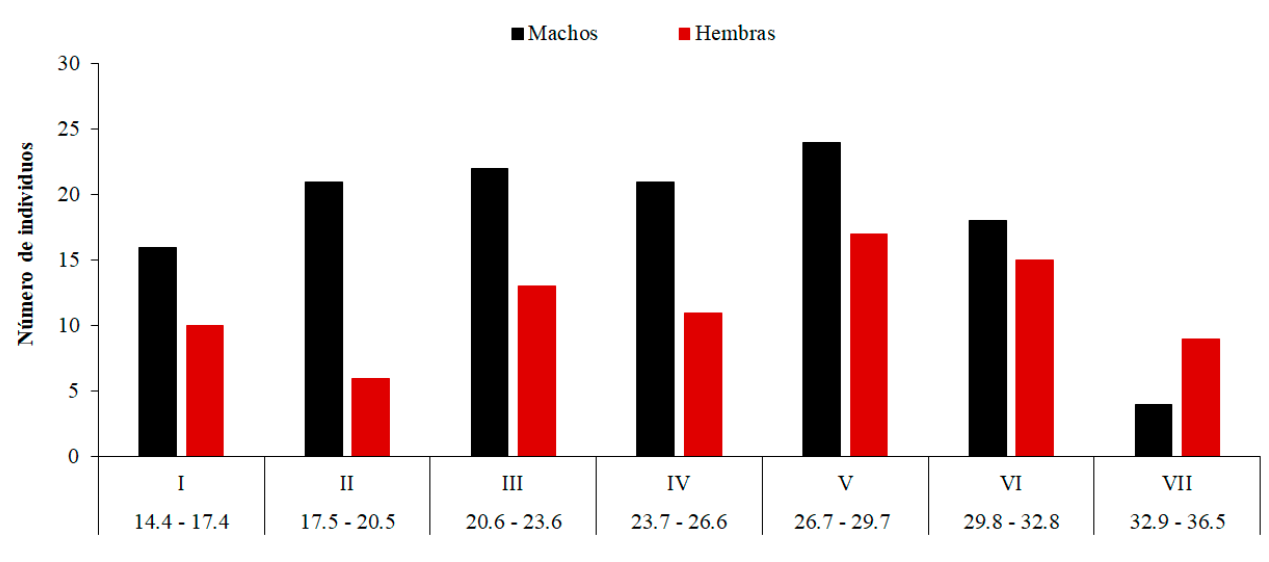

Tallas (cm)

Figura 3. Distribución de frecuencias absolutas por clase de longitud para machos y hembras de Plagioscion magdalenae en el embalse El Guájaro, bajo Magdalena, Colombia.

Figure 3. Distribution of absolute frequencies by length class for males and females of Plagioscion magdalenae in El Guájaro reservoir, lower Magdalena, Colombia.

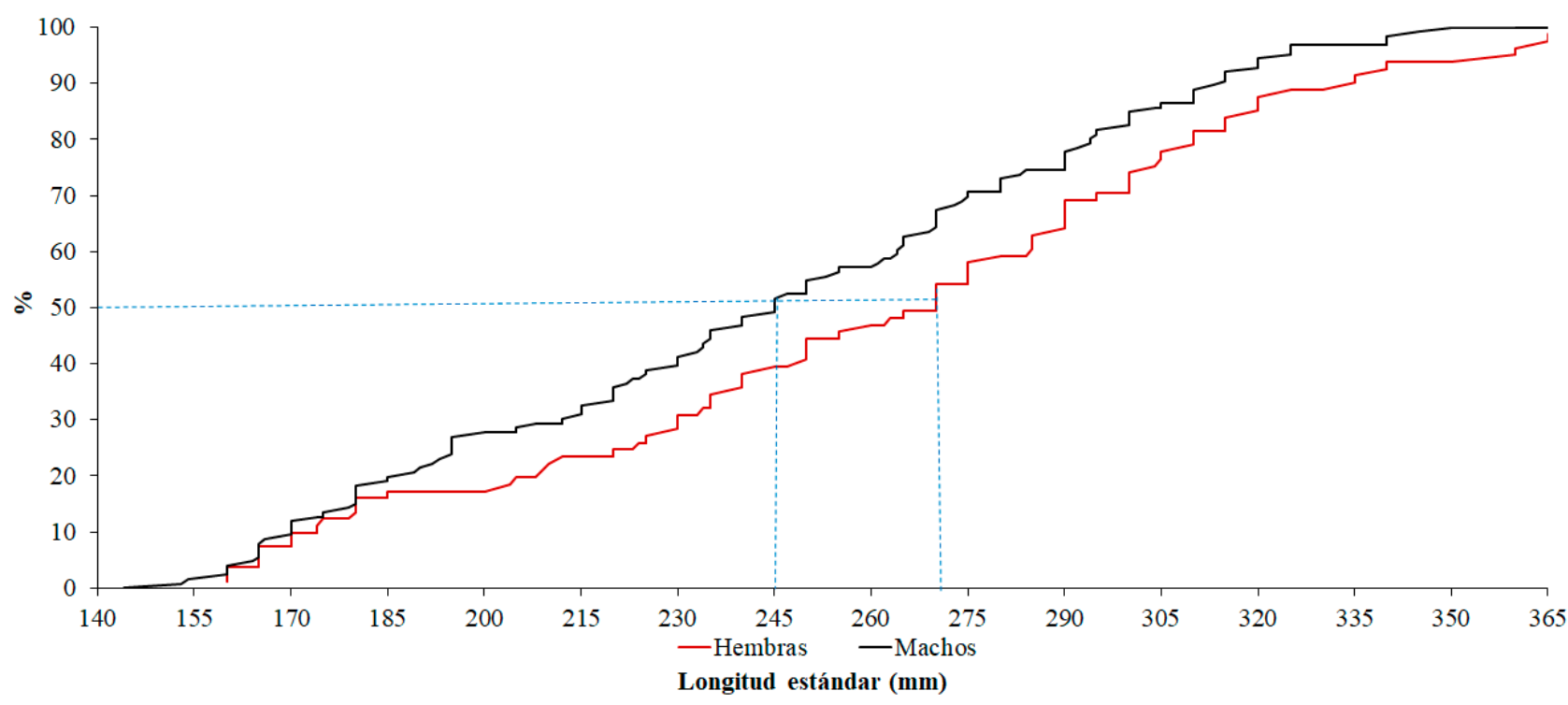

Figura 4. Talla de madurez sexual para machos y hembras de Plagioscion magdalenae en el embalse El Guájaro, bajo Magdalena, Colombia.

Figure 4. Size at sexual maturity for males and females of Plagioscion magdalenae in El Guájaro reservoir, lower Magdalena, Colombia.

El factor de condición K osciló entre 0.9 y 3.1, que se adecua para crecimiento alométrico, e indicó que la especie tiene una buena condición. Las hembras estaban más robustas que los machos; en hembras, los valores mínimos y máximos se registraron en bajas lluvias (mayo y junio) $(\mathrm{K}=1.2, \mathrm{DE}=0.6$ y $\mathrm{K}=4.4, \mathrm{DE}=0.9)$, respectivamente. En machos, el registro más bajo fue en bajas lluvias (enero) $(\mathrm{K}=0.6, \mathrm{DE}=0.8)$, mientras que el registro más alto fue en altas lluvias (diciembre) (K $=2.8, \mathrm{DE}=1.2)($ Figura 5). 


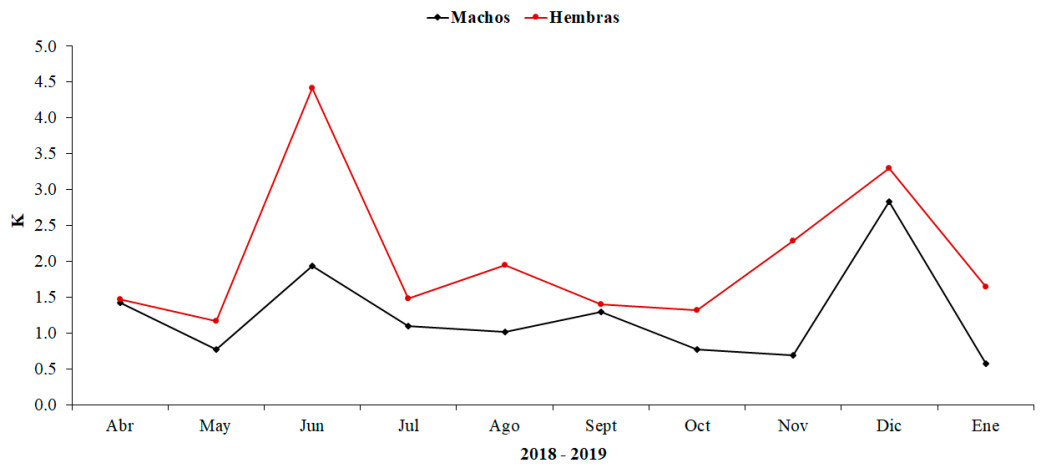

Figura 5. Factor de condición (K) para machos y hembras de Plagioscion magdalenae entre abril de 2018 a enero de 2019 , en el embalse El Guájaro, bajo Magdalena, Colombia.

Figure 5. Condition factor (K) for males and females of Plagioscion magdalenae between April 2018 and January 2019, in El Guájaro reservoir, lower Magdalena, Colombia.

La relación gonadosomática (RGS) en machos varió durante los períodos del pulso de inundación, con registros mínimos durante aguas subiendo $(0.26 \mathrm{y}$ $0.27)$ y máximos durante las aguas altas $(0.39$ y 0.48$)$ y aguas bajas ( 0.35 y 0.36). Los valores de RGS más altos para hembras se presentaron tanto en altas (noviembre: 1.43) como en bajas lluvias (junio: 1.29), seguidos por una disminución abrupta en aguas subiendo (septiembre y octubre: 0.36 y 0.43 , respectivamente). Los registros bajos de RGS, para machos y hembras corresponden al momento de transición de época seca a lluviosa (julio) y altas lluvias (septiembre-octubre); estos, a su vez constituyen los dos picos principales en los cuales la población lleva a cabo su desove, más un pico secundario en aguas altas (diciembre) (Figura 6).

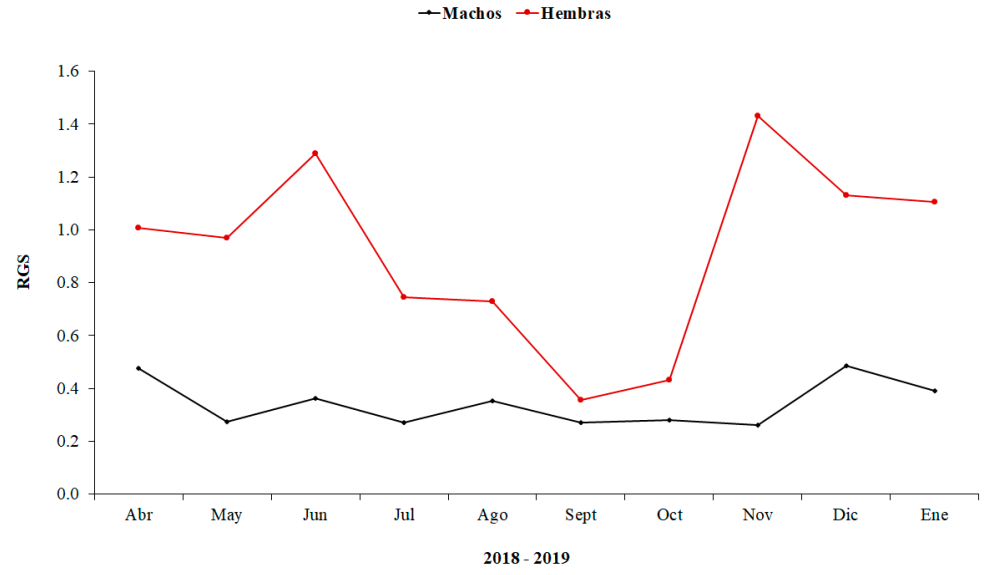

Figura 6. Relación gonadosomática (RGS) para machos y hembras de Plagioscion magdalenae entre abril de 2018 a enero de 2019, en el embalse El Guájaro, bajo Magdalena, Colombia.

Figure 6. Gonadosomatic relationship (GSR) for males and females of Plagioscion magdalenae between April 2018 and January 2019 , in El Guájaro reservoir, lower Magdalena, Colombia.

La fecundidad se estimó en 38 ejemplares maduros con tallas entre 20.4 y $36.5 \mathrm{~cm}$; estos individuos fueron capturados durante todos los meses de muestreo, excepto octubre. Se estimaron en promedio 1909 ovocitos por hembra (588-3231; $\mathrm{DE}=1300.3)$ en aguas bajando, 1590 (319-3 560; $\mathrm{DE}=1405.9)$ en aguas bajas, 2435 (443-4786; $\mathrm{DE}=1228.0)$ en aguas subiendo y 2132 ovocitos (200$7615 ; \mathrm{DE}=2049.6$ ) durante aguas altas. En cuanto a la 
fecundidad relativa (huevos/gramos de peso), P. magdalenae tuvo $6 \pm 7 \mathrm{~h} / \mathrm{g}$ en aguas bajando, $5 \pm 4 \mathrm{~h} / \mathrm{g}$ en aguas bajas, $7 \pm 6 \mathrm{~h} / \mathrm{g}$ en aguas subiendo y $5 \pm 4 \mathrm{~h} / \mathrm{g}$ en aguas altas. Los ovocitos presentaron en general un diámetro de $0.288 \mathrm{~mm}(0.14-0.34 \mathrm{~mm} ; \mathrm{DE}=0.05)$ para las bajas lluvias y $0.290 \mathrm{~mm}(0.14-0.38 \mathrm{~mm} ; \mathrm{DE}=0.06)$ en altas lluvias.

\section{Discusión}

La alometría positiva $(b>3)$ para la relación longitud/peso de P. magdalenae concuerda con lo descrito por Barbosa et al. (2010) para la bahía de Marajó en Brasil, determinando que esta relación tiene importantes aplicaciones en términos de biología pesquera. Según Fonteles (1989), la alometría positiva es posiblemente influenciada por la depredación de otros organismos, sirviendo como mecanismo de defensa, ya que se produce un aumento de tamaño con un mínimo gasto de energía. Además, también puede atribuirse a una alta disponibilidad de alimentos que surgen en estos cuerpos de aguas inundables y de zonas de cría.

El factor de condición $K$, varió a lo largo de los meses de muestreo. Este indicó que las hembras eran más robustas que los machoslo cual tiene relación con la demanda energética que tienen las hembras en los procesos fisiológicos y reproductivos, tales como la acumulación de vitelo durante la fase de maduración de las gónadas, la migración a las zonas de desove y el propio proceso de desove, los cuales requieren reservas energéticas adicionales en las hembras en relación con los machos (Monteiro et al., 2007). El descenso del factor de condición (K) en ejemplares en los meses de julio y septiembre se puede considerar como indicador de la liberación de ovocitos o esperma, información que se corrobora con el RGS (Weatherley, 1972; Román-Valencia et al., 2006). Sin embargo, podría no ser así, como se observa en el mes de diciembre, en el cual, según el RGS, se da un proceso reproductivo, pero se mantiene un grado de robustez alto en los individuos; esto podría explicarse con que este mes coincide con el período de aguas altas y se conoce que la disponibilidad de alimento en éste aumenta considerablemente con respecto a los otros períodos. Además, nuestros registros permiten inferir condiciones nutricionales favorables para la especie y una alta oferta alimentaria, ya que son superiores a 1.5 (Ibarra-Trujillo y García-Alzate, 2017).

La variación en el factor de condición estuvo acorde a los valores del RGS, con valores más altos durante los períodos secos. Esto sugiere un buen estado de alimentación del pez, como preparación para la reproducción y los más bajos durante los momentos en que se llevan a cabo eventos reproductivos. Al parecer, esta especie acumula reservas corporales energéticas, utilizadas para procesos como la migración, la maduración gonadal y el desove, como se describió también para su congénere P. squamosissimus (Carvalho et al., 2017).

La proporción global de sexos de $P$. magdalenae fue de 1:1.6, sin diferencias significativas con la proporción $1: 1$, que es lo que se esperaría en poblaciones naturales. Esto contrasta con lo reportado para la misma especie hace más de 15 años en el embalse El Guájaro y la Bahía de Marajó (Brasil), donde la proporción fue mayor en machos con respecto a las hembras (Bayuelo y Sanz, 2003; Barbosa et al., 2010). Sin embargo, en el estudio se evidenció que los machos predominan en todos los meses, excepto en los de lluvias, cuando predominan las hembras, lo cual puede estar asociado a la facilidad de ser capturadas, debido al peso de sus gónadas (Gurgel, 2004). De igual manera, para P. squamosissimus en un área de protección ambiental al noreste de Brasil, se observó una proporción igual de machos y hembras (Carvalho et al., 2017).

La talla media de maduración sexual observada en este estudio se encuentra por debajo de la talla media de captura establecida por la Autoridad Nacional de Acuicultura y Pesca_AUNAP (2013), que es de $30 \mathrm{~cm}$ de LE, lo que significa que la TMC está bien estipulada. Sin embargo, se registró que se están capturando individuos por debajo de la TMC, el $77.8 \%$ de los individuos registrados en el estudio, lo cual implica una clara presión sobre la población de juveniles, impidiendo que lleguen a reproducirse antes de ser capturados, y llevando a la especie a tener problemas de repoblación. Por otro lado, en los peces, en general, los machos tienden a madurar antes y con un tamaño menor, mientras que las hembras maduran más tarde y tienden a invertir relativamente más recursos en su reproducción (Bromley, 2003). Esto se registra para muchos de los sciaénidos marinos (Santos-Martínez y Acero, 1991; Lowerre et al., 1996; Torres-Castro et al., 1999; Hutchings et al., 2006). Por otro lado, para los de agua dulce, los datos concuerdan con lo registrado para la misma especie por Barbosa et al. (2010), quienes encontraron que los machos maduraban primero que las hembras (269 y $305 \mathrm{~mm}$, para machos y hembras, respectivamente). Sin embargo, para su congénere $P$. squamosissimus se determinó que las hembras maduraban primero que los machos (Barbosa et al., 2012). 
Por otro lado, se han registrado en el embalse individuos cercanos a $70 \mathrm{~cm}$ de LT, casi el doble de los individuos registrados en este estudio, pudiendo estar asociado a la presión pesquera y las condiciones ambientales desfavorables, que pueden forzar a los individuos a tener una maduración temprana para reducir el riesgo de morir antes de reproducirse (Wootton, 1999). Además, la reducción de tamaño de la población resulta en un aumento en la disponibilidad de alimento, ocasionando la aceleración del proceso de maduración, como consecuencia del incremento en la tasa de crecimiento (Vazzoler, 1996).

Plagioscion magdalenae es una especie asincrónica, que tiene desoves múltiples o parcelados durante el año, lo cual se corrobora con los registros de individuos con diferente grado de desarrollo gonadal en todos los meses de muestreo, como ya había sido observado por Bayuelo y Sanz (2003) para la misma especie, y como se ha reportado en el Amazonas para P. squamosissimus y en la Ciénaga Grande de Santa Marta para Bairdiella ronchus, para los cuales se determinó que el desove puede ocurrir durante todo el año (Torres-Castro et al., 1999; Salinas y Agudelo, 2000). Para el caso de P. magdalenae y sus congéneres, este tipo de estrategia reproductiva de desove puede ser para mantener una actividad de reproducción continua de la especie o para sobrevivir a la competencia inter e intraespecífica, además de la presión pesquera local y condiciones abióticas no favorables. Por otra parte, este desove es típico de peces que viven en aguas tranquilas, como lagos o embalses (Bayuelo y Sanz, 2003; Carvalho et al., 2017). En los desoves parcelados, los ovocitos maduran en lotes y son expulsados en intervalos de tiempos distintos, marcando una estacionalidad en estos (Araujo, 2009), reduciendo así la competencia entre la descendencia por alimento y refugio (Godinho, 2007) y permitiendo mayores posibilidades de asentamiento de la especie.

Bayuelo y Sanz (2003) detectaron hembras desovadas en los meses de junio y diciembre, difiriendo de lo encontrado en este estudio, en el que no se obtuvieron hembras en período de desove. Esto está relacionado al hecho de que estos peces desovan en la vegetación flotante que se encuentra desde las orillas hacia el centro del embalse en época de lluvias, lo cual dificulta la captura con las técnicas de pesca utilizadas en las expediciones. Por otro lado, con los valores de RGS se determinan los principales picos reproductivos de la especie, que concuerdan con las temporadas de transición de época seca a lluvias (julio) y altas lluvias (septiembre-diciembre), cuando sube el caudal del río y, por ende, el nivel del agua del embalse. Esto contrasta con lo reportado para P. squamosissimus en el Amazonas, donde el principal evento reproductivo se da en la transición de aguas en descenso al período seco del río, pero es similar a lo registrado en el Orinoco colombiano, donde se da un mayor porcentaje de individuos maduros en el período de inicio de las lluvias y el aumento del caudal del río (Santos et al., 2006). Esto muestra la importancia de los regímenes hidrológicos en la biología y ecología del género Plagioscion.

Plagioscion magdalenae presenta una baja fecundidad comparada con otras especies del género: $P$. squamosissimus, que tiene una elevada fecundidad (22 610-143 337 ovocitos en Brasil, 371025 ovocitos en Venezuela) (Lasso et al., 1998; Felix, 2008; Carvalho et al., 2017). Sin embargo, estos valores bajos de fecundidad (absoluta y relativa) pueden estar relacionados con la captura de individuos pequeños por parte de los pescadores, por debajo o cercanos a la talla media de madurez sexual, pues para algunas especies de peces existe una relación directa entre el tamaño de la hembra y el número de ovocitos que esta puede madurar, siendo común que las hembras pequeñas presenten fecundidades bajas (Nakatani et al., 2001; Valdiviezo-Rivera et al., 2016). Además, la fecundidad es particular a la edad del pez y, en algunos casos, a su talla (Vazzoler, 1996; Murua et al. (2003), $\mathrm{y}$, como se mencionó anteriormente, $P$. magdalenae no está alcanzado ni está cercana a los registros de longitud máxima para el embalse y la cuenca del Magdalena.

La pácora registró un tamaño promedio de ovocitos menor a $P$. squamosissimus $(0.56 \mathrm{~mm})$ pero mayor a $B$. ronchus $(0.19 \mathrm{~mm})$ (Torres-Castro et al., 1999; Barbosa et al., 2012). Los datos de fecundidad registrados en este artículo sugieren una relación con las tallas de las hembras; sin embargo, sería pertinente verificar en estudios posteriores si esos valores obedecen a presiones antrópicas o ambientales.

Los aspectos reproductivos evaluados en este artículo proporcionan bases para la conservación de la especie en el bajo Magdalena, y evidencian la necesidad de realizar estudios de la afectación de los procesos reproductivos a partir de actividades antrópicas como la ganadería, agricultura y descarga de aguas residuales, que son comunes en El Guájaro.

\section{Agradecimientos}

Agradecemos al programa de Biología y Grupo de Investigación Biodiversidad del Caribe Colombiano 
y Estudios en Sistemática y Conservación de la Universidad del Atlántico, a la Vicerrectoría de Investigaciones y la Facultad de Ciencias Básicas, por el apoyo logístico y financiero, convocatoria interna formación de estudiantes a nivel de pregrado. A la Gobernación del Atlántico por parte de la financiación en marco del proyecto regalías: "Estado actual de los recursos hidrobiológicos de las ciénagas del Sur del Atlántico y subregión canal del Dique" código 0103*2013*000009. También agradecemos a Donald C. Taphorn por sus aportes en el abstract.

\section{Referencias}

Álvarez-León, R. \& Jiménez-Segura, L. F. (2012). Plagioscion magdalenae. En Mojica, J. I., Usma, J. S., Álvarez-León, R. \& C. A. Lasso. (Eds). Libro rojo de peces dulceacuícolas de Colombia 2012. (Pp: 112). Instituto de Investigación de Recursos Biológicos Alexander von Humboldt, Instituto de Ciencias Naturales de la Universidad Nacional de Colombia, WWF Colombia y Universidad de Manizales, Colombia.

Araujo, R. B. (2009). Desova e fecundidade em peixes de água doce e marinhos. Revista de Biologia e Ciências da Terra, 9(2), 24-31.

Araya, P., Hirt, L. \& Flores, S. (2003). Biología reproductiva y crecimiento de Pimelodus clarias maculatus (Lac. 1803) (Pisces, Pimelodidae) en la zona de influencia del embalse Yacyretá. Ecologia Austral, 13(1), 83-95.

Autoridad Nacional de Acuicultura y Pesca. (2013). Resolución 409 de 25 de abril de 2013.

Barbosa, N., Rocha, R. M. \& Frédou, L. (2010). Reproductive biology of Plagioscion magdalenae (Teleostei: Sciaenidae) (Steindachner, 1878) in the bay of Marajo, Amazon Estuary, Brazil. Neotropical Ichthyology, $8(2), 333-340$

https:/ / doi.org/10.1590/S1679-62252010000200012

Barbosa, N. D., Rocha, R. M. \& Lucena Frédou, F. (2012). The reproductive biology of Plagioscion squamosissimus (Heckel, 1840) in the Pará River estuary (Amazon Estuary). Journal of Applied Ichthyology, 28(5), 800-805.

https://doi.org/10.1111/j.1439-0426.2012.02040.x

Bayuelo-Espitia, V. \& Sanz-Ochotorena, A. (2003). Morfología externa y ciclo ovárico del pez Pacora $(P$. magdalenae) en el Embalse El Guájaro, Colombia. Revista Biología, 17(1), 26-30.

Bromley, P. J. (2003). The use of market sampling to generate maturity ogives and to investigate growth, se- xual dimorphism and reproductive strategy in central and south-western North Sea sole (Solea solea L.). ICES Journal of Marine Science, 60(1), 52-65.

https:/ / doi.org/10.1006/jmsc.2002.1318

Caraballo, P. (2009). Efecto de tilapia Oreochromis niloticus sobre la producción pesquera del embalse El Guájaro Atlántico-Colombia. Revista MVZ Córdoba, 14(3), 1796-1802.

https:/ / doi.org/10.21897/rmvz.339

Cardoso, A. dos S., Santos, N. B., De Almeida, Z. da S., Neta, R. \& Cantanhêde, L. G. (2018). Reproductive biology of king weakfish, Macrodon ancylodon (Perciformes, Sciaenidae) from the northeastern coast of Brazil. Revista de Biología Marina y Oceanografia, 53(1), 95-104.

https:/ / doi.org/10.4067/s0718-19572018000100095

Carvalho, I. F., Cantanhede, L. G., Santos, N. B., Carvalho-Neta, R. N. F. \& Almeida, Z. (2017). Biología reproductiva de Plagioscion squamosissimus (Pisces, Sciaenidae) em uma área de proteção ambiental do nordeste do Brasil. Boletim do Instituto de Pesca, 43(2), 243-256. https:/ / doi.org/10.20950/1678-2305.2017v43n2p243

Casatti, L. (2005). Revision of the South American freshwater genus Plagioscion (Teleostei, Perciformes, Sciaenidae). Zootaxa, 1080, 39-64.

https:/ / doi.org/10.11646/ zootaxa.1080.1.4

Chaves, P. \& Vazzoler, G. (1984). Aspectos biológicos dos peixes amazônicos. II. Anatomia microscópica de ovários, escala de maturidade e tipo de desova das espécies do gênero Semaprochilodus. Revista Brasileira de Biologia, 44(3), 347-359.

CRA, Ministerio del Medio Ambiente, Banco Interamericano de Desarrollo \& CARDIQUE. (2002). Plan de Manejo Ambiental del complejo de ciénagas El Totumo, El Guájaro, y El Jobo en la ecorregión estratégica del Canal del Dique (Convenio No. 201680). (Informe técnico). Ministerio del Medio Ambiente.

Criscuolo, E. (2005). Bases Fisiológicas de la Reproducción en Peces Tropicales. En Daza P., Landines M. \& Sanabria, A. (Eds.), Reproducción de peces en el trópico. (Pp. 23-27). Universidad Nacional de Colombia.

Felix, R. T. S. (2008). Atividade reprodutiva de Plagioscion squamosissimus (Heckel, 1840) (Actinopterydii, Perciformes), no Reservatório de Pedra, Rio de Contas, Bahia. (Trabajo de grado). Universidade Federal Rural de Pernambuco, Departamento de Pesca e Aquicultura.

Fonteles Filho, A. A. (1989). Recursos pesqueiros: biologia e dinámica populacional. (Informe técnico). Impresa Oficial do Ceará.

Froese, R. (2006). Cube law, condition factor and weight-length relationships: History, meta-analysis 
and recommendations. Journal of Applied Ichthyolo$g y, 22,241-253$.

https:/ / doi.org/10.1111/j.1439-0426.2006.00805.x

García-Alzate, C. A., Gutiérrez L. C. \& De la Parra, A. (2016). Embalse de El Guájaro: Diagnostico ambiental y estrategias de rehabilitación. En Alvarado, M. (Ed.). Sur del Atlántico una oportunidad. (Pp: 148-181). Fundación Promigas.

García-Alzate, C., Do Nascimiento, C., Villa-Navarro, F. A., García-Melo, J. E. \& Herrera-R., G. (2020). Diversidad de peces de la cuenca del río Magdalena, Colombia. En Jiménez-Segura, L. \& C. A. Lasso (Eds.). XIX. Peces de la cuenca del río Magdalena, Colombia: diversidad, conservación y uso sostenible. Pp: 85-113. Serie Editorial Recursos Hidrobiológicos y Pesqueros Continentales de Colombia. Instituto de Investigación de Recursos Biológicos Alexander von Humboldt.

Godinho, H. P. (2007). Estratégias reprodutivas de peixes aplicadas à aquicultura: bases para o desenvolvimento de tecnologias de produção. Revista Brasileira de Reprodução Animal, 31(3), 351-360.

Gurgel, H. de C. B. (2004). Estrutura populacional e época de reprodução de Astyanax fasciatus (Cuvier) (Characidae, Tetragonopterinae) do Rio Ceará Mirim, Poço Branco, Rio Grande do Norte, Brasil. Revista Brasileira de Zoologia, 21(1), 131-135.

https:// doi.org/10.1590/s0101-81752004000100022

Hutchings, K., Griffiths, M. H. \& Field, J. G. (2006). Regional variation in the life history of the canary drum Umbrina. Fisheries Research, 77, 312-325. https:/ / doi.org/10.1016/j.fishres.2005.10.011

Ibarra-Trujillo, E. J. \& García-Alzate, C. A. (2017). Ecología trófica y reproductiva de Hemibrycon sierraensis (Characiformes: Characidae) pez endémico del río Gaira, Sierra Nevada de Santa Marta, Colombia. Revista de Biología Tropical, 65(3), 1033.

https:/ / doi.org/10.15517/rbt.v65i3.29439

Lasso-Alcalá, O. M., Lasso-A, C. A. \& Señaris, J. C. (1998). Aspectos de la biología y ecología de la curvinata Plagioscion squamosissimus (Heckel, 1840) (Pisces: Sciaenidae), en los Llanos inundables del estado Apure, Venezuela. Sociedad de Ciencias Naturales La Salle, 58(149), 3-33.

Lasso, C. A., De Paula Gutiérrez, F., Morales-Betancourt, M. A., Agudelo, E., Ramírez-Gil, H. \& Ajiaco-Martínez, R. E. (Eds). (2011) II. Pesquerías continentales de Colombia: cuencas del Magdalena-Cauca, Sinú, Canalete, Atrato, Orinoco, Amazonas y vertiente del Pacífico. Serie Editorial Recursos Hidrobiológicos y Pesqueros Continentales de Colombia. Instituto de Investigación de los Recursos Biológicos Alexander von Humboldt.
Lowerre-Barbieri, S. K., Chittenden, M. E., Barbieri, L. R. (1996). The multiple spawning patterns of weakfish in the Chesapeake Bay and Middle Atlantic Bight. Journal Fish Biology, 48, 1139-1163.

https:/ / doi.org/10.1111/j.1095-8649.1996.tb01811.x Maldonado-Ocampo, J. A., Vari, R. P. \& Usma, J. S. (2008). Checklist of the freshwater fishes of Colombia. Biota Colombiana, 9(2), 143-237.

Merino, M. C. (2018). Acuicultura en Colombia. (Informe técnico). Dirección Técnica de Administración y Fomento, Autoridad Nacional de Acuicultura y Pesca - AUNAP.

Monteiro, V., Benedito, E. \& Domingues, W. M. (2007). Efeito da estratégia de vida sobre as variações no conteúdo de energia de duas espécies de peixes (Brycon hilarii e Hypophthalmus edentatus), durante o ciclo reprodutivo. Acta Scientiarum - Biological Sciences, 29(2), 151-159.

https:/ / doi.org/10.4025/actascibiolsci.v29i2.521

Morales, J. \& García-Alzate, C. A. (2016). Estructura trófica de los peces en arroyos del Corral de San Luis, cuenca del Bajo Magdalena, Caribe, Colombia. Revista de Biología Tropical, 64, 715-732.

https:// doi.org/10.15517/rbt.v64i2.18781

Murua, H., Kraus, G., Saborido-Rey, F., Witthames, P., Thorsen, A. \& Junquera, S. (2003). Procedures to estimate fecundity of marine fish species in relation to their reproductive strategy. Journal of Northwest Atlantic Fishery Science, 33, 33-54. https:// doi.org/10.2960/J.v33.a3

Nakatani, K., Agostino, A., Bialetzki, A., Vanderlei, S., Cavicchioli, P., Makrakis, M. \& Pavanelli, C. (2001). Ovos e larvas de peixes de agua doce: Desenvolvimento e manual de identificação. EDUEM.

Pareja-Carmona, M., Jiménez-Segura, L. \& Ochoa-Orrego, L. (2014). Variación espacio-temporal de las larvas de tres especies de peces migratorios en el cauce del río Magdalena (Colombia), durante el ciclo hidrológico 2006-2007. Actualidades Biológicas, 36(100), 33-38.

Ricker, W. E. (1968). Methods for assessment of fish production in fresh waters. Oxford: Blackwell Scientific. Journal of Wildlife Management, 33(3), 725.

Román-Valencia, C., Hernández, J. H. \& Samudio, H. F. (2006). Sobre ecologia de Characidium caucanum (Pisces: Crenuchidae) en el alto Rio Cauca, Colombia. Dahlia, 9, 33-42.

Salinas, Y. \& Agudelo, E. (2000). Peces de importancia económica de la cuenca amazónica colombiana. Serie, Estudios Regionales de la Amazonia Colombiana, Programa de Recursos Hidrobiológicos. Instituto Amazónico de Investigaciones Científicas, SINCHI. 
Santos-Martínez, A. \& Acero, A. (1991). Fish community of the Ciénaga Grande de Santa Marta (Colombia): Composition and zoogeography. Ichthyological Exploration of Fresh Waters, 2(3), 247-263.

Santos, G., Ferreira, E. \& Zuanon, J. (2006). Peixes comerciais de Manaus. INPA.

Simpson, A. C. (1951). The fecundity of the plaice. Fishery investigations London, 17(2), 27.

Sokal, R. R. \& Rohlf, F. J. (1987). Introduction to Biostatistics. The Mathematical Gazette, 72(460), 150. https:/ / doi.org/10.2307/3618952

Sokal, R. R. \& Rohlf, F. J. (1995). Biometry. W.H. Freemand. Souza Costa, F. J., Coutinho, D. P. \& Wosiacki, W. B. (2019). Phylogenetic relationship of the species of Plagioscion Gill, 1861 (Eupercaria, Sciaenidae). Zoology Elsevier, 132, 41-56. https:/ / doi.org/10.1016/j.zool.2019.01.001

Steindachner, F. (1878). Zur Fischfauna des Magdalenen-Stromes. Anzeiger der Akademie der Wissenschaften in Wien, 15, 88-91.
Torres-Castro, L., Santos-Martínez, A. \& Acero, A. (1999). Reproducción de Bairdiella ronchus (Pisces: Sciaenidae) en la Ciénaga Grande de Santa Marta, Caribe colombiano. Revista de Biología Tropical, 47(3), 553-560. https:/ / doi.org/10.15517/rbt.v47i3.19205

Valdiviezo-Rivera, J., Terneus, E., Vera, D. \& Urbina, A. (2016). Análisis de producción gonadal del pez Grundulus quitoensis Román-Valencia, Ruiz-C. \& Barriga, 2005 (Characiformes: Characidae) en la laguna altoandina "El Voladero" provincia El Carchi, Ecuador. Biota Colombiana, 17(2), 89-97. https:/ / doi.org/10.21068/c2016.v17n02a07

Vazzoler, A. E. (1996). Biología da reproduçâo de peixes teleósteos: teoría e práctica. EDUEM.

Wenner, A. M. (1972). Sex ratio as a function of size in marine Crustacea. American Naturalist, 106, 321-350. https:/ / doi.org/10.1086/282774

Weatherley, A. H. (1972). Growth and Ecology of Fish Populations. London Academic Press.

Wootton, R. (1999). Ecology of Teleost Fishes. Chapman y Hall. 
René A. Rojas-Luna

Universidad del Atlántico.

Barranquilla, Colombia.

rarojas@est.uniatlantico.edu.co

https://orcid.org/0000-0003-0990-7808

\section{Carlos A. García-Alzate}

Universidad del Atlántico.

Barranquilla, Colombia.

Corporación Universitaria Autónoma del Cauca, Popayán, Colombia.

carlosgarciaa@mail.uniatlantico.edu.co

https:/ / orcid.org/0000-0002-8527-0661
Aspectos de la reproducción de Plagioscion magdalenae (Pisces: Sciaenidae) en el embalse El Guájaro, bajo Magdalena, Colombia

Citación: Rojas-Luna, R. A. y García-Alzate, C. A. (2022). Aspectos de la reproducción de Plagioscion magdalenae (Pisces: Sciaenidae) en el embalse El Guájaro, bajo Magdalena, Colombia. Biota Colombiana, 23(1), e971.

https:/ / doi.org/10.21068/2539200X.971

Recibido: 19 de mayo 2021

Aceptado: 6 de agosto 2021 